BULLETIN OF THE

AMERICAN MATHEMATICAL SOCIETY

Volume 81, Number 3, May 1975

\title{
THE BOOLEAN ALGEBRA OF LOGIC
}

\author{
BY WILLIAM HANF 1
}

Communicated by Alistair Lachlan, January 23, 1975

A general method of constructing finitely axiomatizable theories is sketched. It is shown that every recursively enumerable Boolean algebra is isomorphic to the Boolean algebra of sentences of some finitely axiomatizable theory. More complete details of the proof will appear in a forthcoming monograph by William Hanf, Dale Myers, and Roger Simons. This verifies Conjecture I of Hanf [2] that every axiomatizable theory is recursively isomorphic to a finitely axiomatizable theory. It solves a problem of [2] by showing that there exists a finitely axiomatizable undecidable theory with countably many complete extensions and shows that Conjecture II is false. Another consequence is that there exists a finitely axiomatizable theory whose Boolean algebra of sentences has an ordered basis of type $\theta$ where $\theta$ is any constructive ordinal. A complete characterization of $\mathfrak{B}_{\langle 2\rangle}$, the Boolean algebra of sentences of first order logic with equality and a single binary predicate, is obtained. These last two results answer problems considered by Tarski in the late 1930's and proposed to the author around 1960.

THEOREM 1. Given any recursively enumerable linear order type $\alpha$, there exists a finitely axiomatizable theory $F$ whose Boolean algebra of sentences has an ordered basis of type $1+\alpha$.

Sketch of Proof. Four two-tape nonwriting Minsky machines (see $[4, \S 1])$ are constructed. Each machine has a halt instruction and a nondeterministic branch instruction in addition to the four instructions for incrementing and decrementing the two tapes. Machine $A$ is a universal machine which can interpret the program of any Minsky machine. It will be used to simulate the operation of machines $B, C$, and $D$. Each of these machines

AMS (MOS) subject classifications (1970). Primary 02J05; Secondary 06A40, $02 \mathrm{G} 15,02 \mathrm{H} 05$.

Key words and phrases. Boolean algebra of sentences, finitely axiomatizable theories, recursively enumerable Boolean algebra, recursively presentable Boolean algebra, order types, ordered basis.

${ }^{1}$ Research supported in part by National Science Foundation Grant GP-38401. 
determines a sequence of 0 's and 1's by executing a series of nondeterministic branch instructions. Machine $B$ halts unless the sequence is all 0 's, machine $C$ halts unless the sequence is all 1's, and machine $D$ halts unless the sequence represents a cut in an ordering of $\omega$ of type $\alpha$.

The language of $F$ contains two binary predicates $R$ and $S$ which are "next to" relations on planes of points which satisfy unary predicates corresponding to tile types of a Robinson tiling (see [5, $\$ 2$ and 3]; additional signals are provided to prevent misalignment along a fault line). The language also contains a $<$ predicate which is used to relate tape-square points above and below the Robinson plane to points on a fault line of the plane which satisfy additional unary predicates corresponding to instructions in the program for machine $A .^{2}$ The axioms for $F$ insure that the number of tape-square points above and below the Robinson plane increase and decrease according to the instructions given by machine $A$, that in any model of $F$ there are exactly three Robinson planes each with intersecting fault lines (marked by a special unary predicate), that the operation of machine $A$ starts off at the three intersections with tape lengths corresponding to coded programs for machines $B, C$, and $D$ respectively, and that machine $A$ never halts.

The Robinson tilings insure that there are no cyclic configurations of points in any model of $F$ and the presence of machines $B$ and $C$ and special techniques employed in programming machine $A$ guaranty that any local configuration of points in a nonstandard Robinson plane of a model is already present in one of the three standard Robinson planes. A lemma similar to Lemma 2.3 of [2] thus insures that any model of $F$ is elementarily equivalent to a model with only the three standard planes and therefore every sentence is equivalent to a Boolean combination of sentences concerning the nondeterministic branches of machine $D$.

THEOREM 2. $\mathfrak{B}_{\langle 2\rangle}$ is pseudo-indecomposable $\mathfrak{B}^{3} \mathfrak{B}_{\langle 2\rangle}$ is isomorphic to the

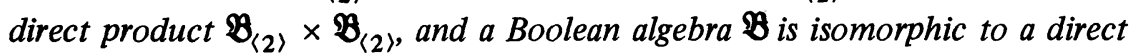
factor of $\mathfrak{B}_{\langle 2\rangle}$ if and only if $\mathfrak{B}$ is recursively enumerable (i.e. $\mathfrak{B}$ is isomorphic

${ }^{2}$ The use of a less-than relation in the direction of the tapes was suggested by Dale Myers who also participated in many useful discussions of earlier versions of the proof.

${ }^{3}$ For Boolean algebra terminology, see [3]. Theorem 2 implies that $\bigotimes_{2}$ is not primitive and that it has factors with ordered basis of type $\omega^{\omega}, \omega+\eta, \omega \cdot \eta_{0}$, $\left(\eta_{0}+\omega \cdot \eta_{0}\right) \cdot \eta_{0}$, etc. Theories with ordered bases of these last two types were found in June 1973 by Myers (using Robinson planes). 
to the quotient algebra of the free Boolean algebra with denumerably many generators modulo an r.e. ideal).

THEOREM 3. $\mathfrak{B}_{\langle 2\rangle}$ has an ordered basis of type $\left(\Sigma_{i \in \omega} \alpha_{i}\right) \cdot \eta_{0}$ where $\alpha_{i}$ is any sequence consisting of r.e. order types (each having a first element) and containing all of them.

Feiner points out in [1] that Conjecture I of [2] together with his result that there are r.e. Boolean algebras that are not recursively presentable implies that $\mathfrak{B}_{\langle 2\rangle}$ is not recursively presentable.

\section{REFERENCES}

1. L. Feiner, Hierarachies of Boolean algebras, J. Symbolic Logic 35 (1970), 365-374. MR 44 \#39.

2. W. Hanf, Model-theoretic methods in the study of elementary logic, Theory of Models (Proc. 1963 Internat. Sympos., Berkeley), North-Holland, Amsterdam, 1965, pp. 132-145. MR 35 \#1457.

3. - Primitive Boolean algebras, Proc. Sympos. in Honor of Alfred Tarski (Berkeley, 1971), Proc. Sympos. Pure Math., vol. 25, Amer. Math. Soc., Providence, R. I., 1974, pp. 75-90.

4. M. Minsky, Recursive unsolvability of Post's problem of "tag" and other topics in theory of Turing machines, Ann. of Math. (2) 74 (1961), 437-455. MR 25 \#3825.

5. R. Robinson, Undecidability and nonperiodicity for tilings of the plane, Invent. Math. 12 (1971), 177-209. MR 45 \#6626.

DEPARTMENT OF MATHEMATICS, UNIVERSITY OF HAWAII, HONOLULU, HAWAII 96822 